\title{
Percutaneous Radiologic Gastrostomy: A 12-Year Series
}

\author{
Franco Perona, Giorgio Castellazzi, Alessandro De luliis, and Laura Rizzo \\ Alliance Medical Italy, Lissone, Italy
}

Background/Aims: Interventional radiologists have played a main role in the technical evolution of gastrostomy, from the first surgical/endoscopical approaches to percutaneous interventional procedures. This study evaluated the results obtained in a 12-year series. Methods: During the period December 1996 to December 2008, 254 new consecutive gastrostomies and 275 replacement procedures were performed in selected patients. All of the cases were treated by a T-fastener gastropexy and tube placement. The procedures were assessed by analyzing indications, patient selection, duration of the procedures, and mortality. Results: All 254 first gastrostomies were successful; replacement procedures were also successfully performed. One $(0.2 \%)$ patient with severe neurologic disorders died after the procedure without signs of procedure-related complications, and seven (1.3\%) major complications occurred (four duodenal lesions with peritoneal leakage, two gastric bleedings, and one gastric lesion). Minor complications were easily managed; three tube ruptures were resolved. Conclusions: This long-term series and follow-up showed that a group of interventional radiologist can effectively provide gastrostomy placement and long-term tube management. Percutaneous gastrostomy is less invasive than other approaches and it satisfies the needs even of high-risk patients. (Gut Liver 2010;4(Suppl. 1):S44-49)

Key Words: Gastrostomy; Percutaneous endoscopic gastrostomy; T-fastener; Percutaneous radiological gastrostomy

\section{INTRODUCTION}

Percutaneous endoscopic gastrostomy (PEG) has be- come the state of the art to achieve a feeding access in patients with neoplastic involvement of the head and neck or oesophagus. However, PEG may fail in patients with stenotic lesions that cannot be passed by an endoscope. Also, obesity, gastric surgery, or other anatomical abnormalities making transillumination of the abdominal wall difficult, may lead to failure of PEG procedures. $^{1}$

As reported in the Literature surgical gastrostomy would be necessary in these cases. Interventional Radiology as become a valid and effective therapeutic alternative to many pathologic conditions and percutaneous radiological gastrostomy (PRG) represents an alternative that avoids both surgery and endoscopy. ${ }^{1}$

Despite encouraging results in literature, this method has not yet gained widespread clinical acceptance. The offer of this promising technique to clinicians of departments for radiation oncology, head and neck surgery, neurology has been enthusiastically taken and they have readily accepted the modality.

The reasons of this approach are related to the need of parenteral nutrition or enteric route for nutrition, to avoid secondary malnutrition. We have to take into account that long-term nasogastric and parenteral devices are not feasible because of an increased risk of gastro-esophageal reflux and subsequent aspiration. ${ }^{2}$

Percutaneous gastrostomy represents a valid alternative, less invasive than surgical approach and more tolerated from patients: it is an artificial entero-cutaneous fistula between stomach and the skin surface, and it can be obtained with endoscopic or radiologic method. ${ }^{3}$

In the last years, PEG has quickly replaced the surgical approach and it is the current standard procedure to obtain a feeding access in these kind of patients. ${ }^{4}$

\footnotetext{
Correspondence to: Franco Perona

Alliance Medical Italy, Piazza G. La Pira 9, Lissone (MI), Italy

Tel: +39-039-46621, Fax: +39-039-4662201, E-mail: franco.perona@alliancemedical.it DOI: $10.5009 /$ gnl.2010.4.S1.S44
} 
The reasons of failure of the endoscopic approach are well known and allow the research of possible alternatives as percutaneous placement of feeding tubes like a valid option to surgery and endoscopy: the interventional approach is easy and fast as well as less invasive as compared to surgery.

Few studies on PRG are focused on extensive series: so, the aim of this study is to analyze our experience in PRG placement with gastropexy in large population focusing on technical difficulties, patients selection and gastrostomy site management and complications.

\section{MATERIALS AND METHODS}

\section{Patients}

From January 1996 to December 2008, 529 patients (229 women, 300 men; mean age, 65 years), referred to our Center, were considered eligible for PRG placement: 254 for first placement, and 275 for replacement.

The clinical indications for first procedure were neurological disease, head and neck cancers; we exclude from our study patients with total gastrectomy, gastric carcinomatosis, extensive gastric varices, critical illness and severe ascites. While, the indications for replacement were tube malfunction or dislodgment or request of the referring physician or patient. ${ }^{4,5}$

Approval to the procedure was obtained from the hospital ethics committee and informed consent obtained from all patients.

\section{Procedure introduction}

The protocol was identical for all the patients on first placement or replacement: before the procedure, complete anamnesis is obtained and a pertinent physical examination assured the appropriateness of gastrostomy (absence of abdominal hernias, or cutaneous scars), and of gastrostomy substitution.

All procedures have been monitored by analyzing indications, patients selection, duration of the procedures, and mortality.

The presence of an anesthesiologist in the angiographic room was required, and the vital parameters were monitored. Technical success was accomplished when the gastrostomy tube was effectively placed into the stomach, and the correct function of the feeding tube was achieved. ${ }^{6}$

\section{Procedure for first placement}

Patients usually were admitted to the hospital for at least 12 hours before the procedure. No sedation was used and no antibiotic were routinely given. The liver and spleen are marked by ultrasound.

The stomach was transorally probed with a $5 \mathrm{~F}$ catheter and a guidewire, and then a nasogastric tube was placed shortly before the procedure to allow air insufflation: stomach was distended with variable volume of air from 500 to $700 \mathrm{~mL}$, using $60 \mathrm{~mL}$ syringe under fluoroscopic control. Immediately before the catheter placement $5 \mathrm{mg}$ of Joshine N-butyl-bromide (Buscopan; Boehringer Ingelheim, Florence, Italy) was injected for gastroparesis in all patients. After sterile cleaning of skin with Pyodine, injection of Xylocane (2\%) was obtained at the site of puncture. $^{2}$

The area in which the gastrostomy tube will be inserted is usually located in the left hypochondrium, 4-8 $\mathrm{cm}$ from median line: a small skin incision is the first step after local anaesthesia and then the stomach is punctured in mid-distal body, to avoid the gastric major arterial branches, with 18-gauge needle. The regular placement of the needle is detected by aspiration of air and flushing with contrast media; after checking the correct position of the needle the T-fastener anchor bar is loaded (Cope Gastrointestinal Suture Anchor; Cook, Bloomington, IN, USA) by pushing coaxially the guidewire.

After that, the needle is removed and the first small caliber introducer is inserted to secure the tract; the anchor bar is then retracted and first suture between anterior abdominal and stomach wall has been completed as the T-fastener mechanism is fixed externally by suture; one or two T-fasteners are usually placed nearby the site of proposed gastrostomy entry. ${ }^{1,3}$

Serial dilatation of percutaneous gastric tract is done with Seldinger technique using dilators up to 12-14 F: finally, gastrostomy polyurethane (Wills Oglesby; Wilson Cook Inc., Bloomington, IN, USA) tube $12 \mathrm{~F}$ is placed, and fixed by means of a loop. ${ }^{3}$

The correct position is checked again by injecting contrast through the tube; the stomach is then decompressed for at least 12 hours following the procedure. T-fasteners will be removed within 5-10 days, under fluoroscopic control.

All patients have a 30-day post-procedure period of observation by the same physician performing the procedure. $^{4}$

\section{Procedure for replacement}

The same technique by insertion, under local anaesthesia, of the guidewire through the tube and then the placement of the optimal feeding tube in the matur tract. Two controls after the procedure and 24 hours later assure the best standard of quality. 


\section{Major complications}

Major complication were defined as those who prolonging the hospital stay, requiring an additional procedure or transfusion. ${ }^{1,2}$

\section{1) Aspiration}

Aspiration resulting in pneumonitis or pneumonia is one of the most frequently reported major complications of PEG placement. Aspiration can occur either during or after the PEG placement procedure. Periprocedure aspiration of oropharyngeal contents occurs in $1 \%$ of patients undergoing PEG tube placement and carries a very high mortality. The level of sedation, frequency of suctioning, and degree of elevation of the head of the bed may all contribute to aspiration risk. Postprocedure aspiration consists of either oropharyngeal contents or refluxed gastric contents and tube feedings. Although a frequently used justification for PEG placement is the prevention of aspiration, this is not supported by the available evidence.

Furthermore, many patients have macroaspiration of gastric contents and tube feedings. Close monitoring of gastric residual volumes and holding feedings when high residuals are encountered may limit aspiration. The presence of an abnormal swallowing evaluation and reflux esophagitis places patients at significantly increased risk for aspiration pneumonia within 1 month of PRG placement.

\section{2) Peritonitis}

Peritonitis is a feared complication of PRG that often carries a high mortality rate. Peritonitis manifests as abdominal pain, fever, and leukocytosis. Causes of peritonitis include removal or displacement of the tube prior to tract maturation, leakage from the PEG puncture site in the stomach, and perforation of another visceral organ. There are no guidelines for the management of peritonitis, but generally patients are treated with broad-spectrum antibiotics.

\section{3) Hemorrhage}

During the procedure hemorrhage may be caused by puncture of gastric wall vessels. The most common cause of hemorrhage post-PEG is due to the ulceration of the gastric mucosa underneath the internal bumper when applied in very tight approximation to the mucosa. Esophageal trauma, gastric erosions, and unrelated peptic ulcer disease are less common etiologies of gastrointestinal bleeding after PRG. Post-PEG hemorrhage is managed similar to other episodes of upper gastrointestinal bleeding.

\section{4) Tube migration and the buried bumper syn- drome}

The buried bumper syndrome is defined as migration of the PEG tube into the gastric wall and the subsequent epithelization of the ulcer site. Buried bumper syndrome often manifests months to years after PEG placement (median duration was 35 months after PEG placement) as abdominal pain; difficulty feeding or flushing the tube; and inability to advance, withdraw, or rotate the tube. Treatment involves removing the tube (which may require upper endoscopy), allowing the tract to close while an alternative method of feeding is established, and then placing a new PRG tube in a different location.

\section{5) Gastrocolocutaneous fistula}

Although colonic perforation during PEG placement is a rare complication, there are possibilities of such complications. The transverse colon is apposed to the greater curvature of the stomach and if the stomach is not well insufflated during placement of the PEG tube, the colon may not be completely displaced out of the field, thus leading to puncture by the gastrostomy tube. Patients who do not manifest signs of obstruction or peritonitis can be managed by tube removal. In most cases, the fistula will close and a second gastrostomy can be performed. If obstruction or peritonitis is present or the fistula does not close despite PEG removal, operative takedown of the fistula is necessary.

\section{6) Wound infection and necrotizing fasciitis}

These complications occur along a continuum, from simple peristomal infections to life-threatening necrotizing fasciitis. Peristomal wound infection is fairly common, occurring in $5-25 \%$ of cases in cohort studies. Antibiotic prophylaxis has been found to be a cost-effective strategy in this setting. Necrotizing fasciitis is a very rare complication and the most aggressive type of PEG-related wound infection. Patients often present with progressive erythema and edema around the site, that then progresses to bullous lesions; septic shock can develop rapidly. Mortality is greater than $50 \%$ in these cases.

\section{7) Inadvertent removal of PEG tube}

Maturation of the PEG tract can occur as early as 1 week after tube placement. Often maturation takes up to 3 weeks given that a majority of patients are severely ill, on corticosteroids, malnourished, and generally manifest poor wound healing. Agitated or delirious patients who inadvertently pull out their PRG tube often can be successfully managed with nasogastric suction and PRG 
replacement. Anecdotally, the PEG tract closes in 24-48 hours when the patient is treated with bowel rest with or without nasogastric suction. Subsequent placement of a PEG tube in a new site is often successful. Signs of peritonitis mandate treatment with antibiotics and a surgical consultation. If a PEG tube is inadvertently removed from a mature tract ( $>3-4$ weeks old), a Foley catheter can be inserted to maintain tract patency, but this should not be attempted if the PEG tract is immature.

\section{Minor complications}

Leakage is a common complaint and a major symptom that causes the patient, family, or caregiver to request a PEG tube change. Factors that predispose to leakage include caustic agents (ascorbic acid) infused via the tube or used to clean the skin around the stoma (hydrogen peroxide) as well as local fungal or bacterial skin infections. Leakage occurring due to tube failure may occur in as many as $25 \%$ of cases at 5 months, and generally requires tube exchange. Leakage from the stoma usually occurs as the stoma dilates over time. Blockage of the tube is also a common problem seen in patients with long-term PEG placement. Silicone tubes had statistically significant higher rates of blockage that correlated with a higher rate of fungal colonization in the tubes. Tube blockage can be minimized by frequent water flushes after feeding, only administering liquid medications or well-ground pills via the tube, and avoidance of materials likely to adhere to the inside of the tube. Blockages can often be removed at bedside either by flushing the tube with warm saline, infusing a phosphate-rich solution, or gently passing an endoscope cleaning brush through the tube.

\section{RESULTS}

\section{Success rate}

Success rate for PRG was $100 \%$ both at first placement both at replacement, and enteral nutrition was started on the second day using nutripump with a specific nutritional protocol. In the 29 patients $(5.4 \%)$ without an indwelling nasogastric tube, the oesophagus was catheterized with a $5 \mathrm{~F}$ catheter in a median time of 3 minutes (range, 1-12 minutes). The median time required for gastropexy was 8 minutes (range, 5-12 minutes) and that for subsequent gastrostomy tube insertion was 9 minutes (range, 6-14 minutes). The median total procedure time was 21 minutes (range, 18-37 minutes).

Fluoroscopy levels were significantly monitored in order to reduce X-ray exposure and the average time was 2.1 minutes (range, 1.3-9 minutes).

\section{Mortality}

Only one patient $(0.2 \%)$ with severe neurologic disorders died because of acute cardiovascular event: there were no signs of correlation with the procedure.

\section{Complications}

In our experience major complications were drastically reduced by careful and state of the art technique, placement in the sterile environment of the interventional suite and long experienced operators. Only seven (1.3\%) major complications occurred: four duodenal lesions with peritoneal leakage, two gastric bleedings and one gastric erosive lesion. Duodenal lesion were managed by retracting the tip of the catheter and by medical therapy. Gastric bleedings were correlated to the tight sutures of the T-fastener in one case and to small gastric varices: both have been successfully managed. The gastric lesion due to decubitus of the indwelling nasogastric tube healed by decompressing the stomach and by medical therapy as well. Twenty-four (4.5\%) minor complications were easily managed: 9 abdominal pain, 5 fever, 7 gastroparesis and 3 tube ruptures, near the external luer lock system.

\section{Follow-up}

All patients underwent contrast examination the day after insertion. The median clinical follow-up was 4.4 months (range, 1-10 months).

Eleven short term malfunction $(2 \%)$ which required to repeat the procedures into the 30-day period were detected; the reason was due to clogged tubes or accidental catheter dislodgement or rupture. All were completed by simple tube exchange (usually for a major caliber tube, 16/18 F) under fluoroscopy, without complications.

\section{DISCUSSION}

The growing experience of the percutaneous method offered to the clinicians a valuable alternative for nutrition support increasingly applied in patients in whom they judged this method preferable to PEG, either in anticipation of failure of endoscopy or in order to avoid mechanical disturbance of recent surgical flaps or grafts in the head and neck region by an endoscopic procedure.

Percutaneous gastrostomy techniques with either radiological or endoscopic guidance have replaced surgical gastrostomies because of the risk of general anaesthesia and the increased morbidity: in general, gastrostomy tubes allows bolus feeding and are easier to place or replace. ${ }^{6}$

This is a single-centre retrospective study designed to evaluated the outcome of patients after PRG placement. 
The outstanding benefit of the radiological technique is the fact that almost every stenosis of the hypopharynx or the oesophagus can be easily probed with hydrophilic guidewires and the respective catheters, which is the essential precondition for the procedure. PRG represents the technique of choice in amyotrophic lateral sclerosis for respiratory distress and lack of anhaestesia. ${ }^{7}$

For fluoroscopic-guided gastrostomy, multiple gastrostomy devices have been described in literature. The initially used Foley catheters have been demonstrated to have an increased morbidity rate including tube leakage, breakage, migration, proximal small bowel obstruction and gastric wall penetration. Most commonly, 10- to 14-F catheters with a Cope Loop retention device have been reported in the literature and were used at our institution. $^{8}$

We performed gastrostomy insertions with the use of T-fastener because of the absence of procedure related deaths, tube extrusion into the peritoneal space: gastropexy devices stabilize the stomach during PRG, provide added security against tract disruption in the first few days following catheter placement, reduce risk of site hemorrhage, help to prevent leakage of ascites around a gastrostomy catheter and is simple to use. ${ }^{3,5,9}$ Such anchors have to be cut 5-10 days post-insertion in order to avoid a foreign body reaction, inflammation, or damage to the gastric mucosa. Peristomal leakages, skin infections, and persistent drainage after removal of the gastrostomy catheters have been attributed to retain $\mathrm{T}$-fastener.

Tube obstruction and dislocation have been considered in connection with the shape of the devices used by Willis and Oglesby. ${ }^{10}$ The use of devices with wider diameters (18-20 F) with internal balloon anchoring systems (already used for PEG) has considerably reduced these complications. $^{11}$

The overall incidence of complications after PEG placement reported in the available literature is in the range of $8-30 \%$, and allows to consider the most serious events requiring treatment occur in approximately $1-4 \%$ of cases.

This list resumes the most serious complications: perforation, intra-abdominal hemorrhaging and peritonitis - occur in $<0.5 \%$ of cases. ${ }^{5}$

In PRG the infection of the entry site is considered the most frequent "minor" complication. The high rate of this complication (around 15\%) is certainly the result of the poor clinical conditions of the patients, but we have to take into account the protocols of managing, inserting the device and keeping aseptic the entry site. It is mandatory to avoid excessive pressure and, consequently, ischemia of the T-fastener system and external fixation system, which might cause the infection. ${ }^{12}$ We have to take under sur- velliance the onset of such complications when comparing the 2 methods of PEG and PRG: in fact comparing the results obtained with 177 PEGs and 193 PRGs, Silas et al. ${ }^{13}$ underlined indicated a major incidence of local infection affecting the radiologic method $(23 \%$ vs $11 \%$; $\mathrm{p}<0.02)$.

Some authors report the a major complications rate ranging from $3-10 \%$ with $\mathrm{PEG}^{6}$ vs $0.5-6 \%$ with $\mathrm{PRG}^{1,5,14}$ Minor complications are rated on $6-33 \%$ with PEG vs $2.9-12 \%$ with PRG. ${ }^{8,9}$ In a further review, Giuliano et al. ${ }^{15}$ in a series of 109 patients subjected to PRG by large caliber tubes (20-24 F) found a rate of minor complications around $5.5 \%$.

These results may be probably correlated to the substantial differences in the techniques of inserting the feeding tubes, the choice of different tube diameters and the use of an array of different anchoring systems as balloon, pig-tail or mushroom. ${ }^{16}$ We are deeply aware of the importance of gastropexy to prevent the accidental insertion of both the guide and the tube into the abdominal cavity, ${ }^{17}$ but it is necessary to enhance the possibility of cutaneous bedsore problems and the onset of local pain and peristomal infection if the traction is so strong to cause ischemic complications and if the T-fasteners are not removed within recommended times (maximum 14 days). ${ }^{18}$ So it is clear that to reduce the onset of complications in the daily practice the technical approach, the operative protocols and the level of experience acquired by the Interventional Group are the keypoints to achieve a better performance.

Based on our experience to date, PEG and PRG are not alternatives but complementary: PEG is still simple and easy to perform and should therefore be considered as a valuable technique, particularly in neurologic pathologies.

In general, the fluoroscopic-guided percutaneous gastrostomy technique shows the advantages of both ease and speediness and the high technical success rate of the radiological approach with the beneficial possibility to exchange the gastrostomy tubes that were traditionally used with endoscopic assistance. However PRG permits identification in patients in whom the colon may lie anterior to the stomach. ${ }^{9}$

One disadvantage may be the smaller caliber of the tube and consequently the greater risk of obstruction: however, the first tube can easily replaced with one of greater caliber after 15 to 30 days. Another disadvantage is the lack of possibility to study gastric internal wall.

In conclusion, percutaneous radiological access is technically feasible in nearly all of those cases in whom an endoscopic attempt is not possible, because of stenotic tumor, or other conditions that prevent transillumination of the abdominal wall. 
The gastrostomy technique combines the radiological approach with the beneficial added value of stable, fast and simple procedure and thereby potentially reduces periprocedural complications and improves nutritional therapy: PRG can be performed safely in a large majority of referred patients.

Interventional radiologists may become familiar with PRG quickly and achieve excellent results. In case of use of balloon-retained feeding catheters for radiological gastrostomy the tube is safer and more stable.

When introducing PRG, close cooperation with gastroenterologists, nurses and dietary assistants is important. The experience described here confirms the value of radiological percutaneous gastrostomy as an alternative to surgical gastrostomy and PEG in patients for whom traditional methods are not the method of choice.

\section{REFERENCES}

1. Dinkel HP, Beer KT, Zbaren P, Triller J. Establishing radiological percutaneous gastrostomy with balloon-retained tubes as an alternative to endoscopic and surgical gastrostomy in patients with tumours of the head and neck or oesophagus. Br J Radiol 2002;75:371-377.

2. Chishty IA, Haider Z, Khan D, Pasha S, Rafiq Z, Akhter W. Percutaneous radiologic gastrostomy: results and complications. J Ayub Med Coll Abbottabad 2006;18:36-39.

3. Thornton FJ, Fotheringham T, Haslam PJ, McGrath FP, Keeling F, Lee MJ. Percutaneous radiologic gastrostomy with and without T-fastener gastropexy: a randomized comparison study. Cardiovasc Intervent Radiol 2002;25: 467-471.

4. Rimon E. The safety and feasibility of percutaneous endoscopic gastrostomy placement by a single physician. Endoscopy 2001;33:241-244.

5. Dewald CL, Hiette PO, Sewall LE, Fredenberg PG, Palestrant AM. Percutaneous gastrostomy and gastrojejunostomy with gastropexy: experience in 701 procedures. Radiology 1999;211:651-656.

6. Wollman B, D'Agostino HB. Percutaneous radiologic and endoscopic gastrostomy: a 3-year institutional analysis of procedure performance. AJR Am J Roentgenol 1997;169: 1551-1553.

7. Chio A, Galletti R, Finocchiaro C, et al. Percutaneous radiological gastrostomy: a safe and effective method of nutritional tube placement in advanced ALS. J Neurol Neurosurg Psychiatry 2004;75:645-647.

8. Hicks ME, Surratt RS, Picus D, Marx MV, Lang EV. Fluoroscopically guided percutaneous gastrostomy and gastroenterostomy: analysis of 158 consecutive cases. AJR Am J Roentgenol 1990;154:725-728.

9. Saini S, Mueller PR, Gaa J, et al. Percutaneous gastrostomy with gastropexy: experience in 125 patients. AJR Am J Roentgenol 1990;154:1003-1006.

10. Wills JS, Oglesby JT. Percutaneous gastrostomy. Radiology 1988;167:41-43.

11. Moller P, Lindberg CG, Zilling T. Gastrostomy by various techniques: evaluation of indications, outcome, and complications. Scand J Gastroenterol 1999;34:1050-1054.

12. Akkersdijk WL, van Bergeijk JD, van Egmond $\mathrm{T}$, et al. Percutaneous endoscopic gastrostomy (PEG): comparison of push and pull methods and evaluation of antibiotic prophylaxis. Endoscopy 1995;27:313-316.

13. Silas AM, Pearce LF, Lestina LS, et al. Percutaneous radiologic gastrostomy versus percutaneous endoscopic gastrostomy: a comparison of indications, complications and outcomes in 370 patients. Eur J Radiol 2005;56:84-90.

14. de Baere T, Chapot R, Kuoch V, et al. Percutaneous gastrostomy with fluoroscopic guidance: single-center experience in 500 consecutive cancer patients. Radiology 1999; 210:651-654.

15. Giuliano AW, Yoon HC, Lomis NN, Miller FJ. Fluoroscopically guided percutaneous placement of large-bore gastrostomy and gastrojejunostomy tubes: review of 109 cases. J Vasc Interv Radiol 2000;11:239-246.

16. Funaki B, Zaleski GX, Lorenz J, et al. Radiologic gastrostomy placement: pigtail- versus mushroom-retained catheters. AJR Am J Roentgenol 2000;175:375-379.

17. Brown AS, Mueller PR, Ferrucci JT Jr. Controlled percutaneous gastrostomy: nylon T-fastener for fixation of the anterior gastric wall. Radiology 1986;158:543-545.

18. Tao HH, Gillies RR. Percutaneous feeding gastrostomy. AJR Am J Roentgenol 1983;141:793-794. 